\title{
MÉTODOS DE AVALIAÇÃO DISCENTE EM UM CURSO DE GRADUAÇÃO BASEADO EM METODOLOGIAS ATIVAS
}

\author{
MÉTODOS DE EVALUACIÓN DE ESTUDIANTES EM UM CURSO DE \\ GRADUACIÓN BASADO EM METODOLOGIAS ACTIVAS
}

\section{METHODS OF STUDENT ASSESSMENT IN AN UNDERGRADUATE COURSE BASED ON ACTIVE METHODS}

\author{
Aline Cabral de OLIVEIRA-BARRETO ${ }^{1}$ \\ Raphaela Barroso GUEDES-GRANZOTTI ${ }^{2}$ \\ Danielle Ramos DOMENIS ${ }^{3}$ \\ Ariane Damasceno PELLICANI ${ }^{4}$ \\ Kelly da SILVA ${ }^{5}$ \\ Rodrigo do Carmo DORNELAS ${ }^{6}$ \\ Carla Patrícia Hernandez Alves Ribeiro CÉSAR ${ }^{7}$
}

RESUMO: O presente estudo tem por objetivo comparar e correlacionar os resultados das avaliações formativa e somativa nas sessões tutoriais e habilidades práticas de um curso de graduação em Fonoaudiologia de uma Universidade pública brasileira. A amostra foi constituída por resultados de avaliações somativas e formativas de sessões tutoriais e habilidades práticas de 42 discentes do II Ciclo do curso de Fonoaudiologia de uma Universidade pública, que utiliza como método de ensino-aprendizagem a metodologia ativa, com a abordagem da aprendizagem baseada em problemas. Foram aceitos apenas os resultados de avaliações de discentes com assiduidade mínima de $75 \%$. Os dados foram tabulados e submetidos a tratamento estatístico com p-valor $\leq$ 0,05 . Foi observado correlação positiva entre as avaliações formativa e somativa, tanto em sessões tutoriais quanto em habilidades práticas. Foram identificados maiores escores para a avaliação formativa, com presença de diferenças significativas $(p<0,01)$. Conclui-se que houve correlação positiva entre as avaliações formativas e somativas em sessões tutoriais e de habilidades, com melhores resultados para a formativa (tanto em sessões tutoriais quanto em habilidades). Neste contexto, o presente estudo demonstra a

${ }^{1}$ Docente do Departamento de Fonoaudiologia. UFS - Universidade Federal de Sergipe. E-mail: alinecabralbarreto@gmail.com

${ }^{2}$ Docente do Departamento de Fonoaudiologia. UFS - Universidade Federal de Sergipe. E-mail: raphaelabgg@gmail.com

${ }^{3}$ Docente do Departamento de Fonoaudiologia. UFS - Universidade Federal de Sergipe. E-mail: drdomenis@gmail.com

${ }^{4}$ Docente do Departamento de Fonoaudiologia. UFS - Universidade Federal de Sergipe. E-mail: ariane.pellicani.ap@gmail.com

${ }^{5}$ Docente do Departamento de Fonoaudiologia. UFS - Universidade Federal de Sergipe. E-mail: kelly.fonoufs@gmail.com

${ }^{6}$ Docente do Departamento de Fonoaudiologia. UFS - Universidade Federal de Sergipe. E-mail: rdgdornelas@gmail.com

${ }^{7}$ Docente do Departamento de Fonoaudiologia. UFS - Universidade Federal de Sergipe. E-mail: carlacesar@globo.com 
necessidade da implementação e/ou valorização de avaliações diárias de caráter formativo no desenvolvimento dos cursos de graduação em fonoaudiologia.

PALAVRAS-CHAVE: Avaliação educacional. Ensino. Aprendizagem baseada em problemas.

RESUMEN: Este estudio tiene como objetivo comparar y correlacionar los resultados de las evaluaciones formativas y sumativas en las sesiones de tutoría y habilidades prácticas y del estudiante en técnicas de terapia del habla de una universidad pública brasileña. La muestra era constituida por los resultados de las evaluaciones sumativas y formativas de tutoriales y habilidades prácticas de 42 alumnos de Ciclo II curso de expresión de una Universidad pública, que utiliza como método de enseñanza aprendizaje de la metodología activa, el aprendizaje basado en el enfoque problemas. Sólo se aceptan los resultados de las evaluaciones de los estudiantes con una asiduidad mínima del $75 \%$. Los datos fueron tabulados y sometidos a análisis estadístico con el valor de $p \leq 0,05$. Se observó una correlación positiva entre la evaluación formativa y sumativa, tanto en sesiones de tutoría y en las habilidades prácticas. Puntuaciones más altas fueron identificados para la evaluación formativa, con la presencia de diferencias significativas $(p<0,01)$. Se concluyó que existía una correlación positiva entre la evaluación formativa y sumativa en tutoriales y sesiones de habilidades, con mejores resultados para la formación (tanto en sesiones de tutoría como habilidades). En este contexto, el presente estudio demuestra la necesidad de implementar y / o recuperación de los informes diarios de formación en el desarrollo de cursos de graduación en terapia del habla.

PALABRAS CLAVE: Evaluación de la educación. Educación. Aprendizaje basado en problemas.

ABSTRACT: This study aims to compare and correlate the results os formative and summative assessments in tutorial sessions and skills practices of an undergraduated degree in Speech Therapy of a Brazilian public University. The sample consisted of results of summative and formative assessments of tutorials and practical skills of 42 students of II Cycle of Speech Therapy course of a Public University, which uses a active methododolgy, approached as based learning problems. The inclusion criterion was the acceptance only the results os student's evaluation with minimuin attendace of $75 \%$. Data were tabulated and submitted to estatistical analyses with p-value $\leq 0.05$. There was a positive correlation between assessments (formative and summative), in Tutorials and Skills. Higher mean scores were identified for the two subunits, in the formative evaluation, with significant differences $(p<0.01)$. Conclusion: There was a positive correlation between the formative and summative assessments in tutorial sessions and skills practices, with better results for the formative (both as tutorial sessions and skills practices). In this context, this study demonstrates the need to implement and/ou valorize of daily formative evaluations in the development of Speech Therapy graduations.

KEYWORDS: Educational measurement. Teaching. Problem-based learning. 


\section{Introdução}

$\mathrm{Na}$ última década, diversos questionamentos surgiram sobre o perfil do profissional graduado na área da saúde, dissociado das reais necessidades do sistema de saúde brasileiro. Neste momento, as instituições de ensino na área da saúde foram convidadas a mudarem sua prática pedagógica, em uma tentativa de se aproximarem da realidade social, com destaque para o Sistema Único de Saúde, para uma prática que valorizasse a integralidade, o cuidado humanizado e a promoção à saúde (MERHY; FEUERWERKER; CECCIM, 2006; MARIN et al., 2010).

Nesse contexto, as metodologias ativas de ensino surgiram tendo como alicerce a autonomia e como objetivo formar sujeitos sociais com competências éticas, políticas, técnicas e dotados de conhecimento, raciocínio, crítica, responsabilidade e sensibilidade para as questões da vida e da sociedade, capacitando-os para intervirem em contextos de incertezas e complexidades. Dentre as várias propostas pedagógicas utilizadas nas metodologias ativas, a aprendizagem baseada em problemas (ABP) é uma delas, que se define no ensino centrado no estudante e baseado em solução de problemas. Nessa proposta os problemas permitem o desenvolvimento dos processos de ensinoaprendizagem e valorizam o aprender a aprender, o estudante deixa o papel de receptor passivo e assume o de agente principal responsável pela sua aprendizagem, redirecionando responsabilidades e oferecendo espaço para o desenvolvimento de sua autonomia, tornando-o capaz de gerenciar a sua formação (BERBEL, 1998; FREIRE, 2006; BORGES et al., 2014; DORNELAS et al., 2014).

As reflexões e discussões sobre as situações-problema a que são expostos, desencadeia no estudante a busca de fatores explicativos e a suposição de soluções para o problema. Desta forma, problematizar significa ser capaz de responder ao conflito intrínseco que o problema traz. Os conteúdos são construídos pelo estudante que precisa reorganizar o material, adaptando-o à sua estrutura cognitiva prévia, para descobrir relações, leis ou conceitos que precisará assimilar; retirando da realidade elementos que conferem significado em direção à aprendizagem (GARCIA MADRUGA, 1996; ZANOTTO; DE ROSE, 2003; CYRINO, TORALLES-PEREIRA, 2004, GUEDESGRANZOTTI et al., 2015).

Tão importante quanto o método de aprendizagem, porém bem menos discutida, é a forma de avaliação desse processo, que não deve ser considerada como uma 
atividade neutra ou meramente técnica, mas sim dimensionada por um modelo teórico de mundo, de ciência e de educação, traduzida em prática pedagógica. A prática de avaliação dos processos de ensino e de aprendizagem deve ocorrer por meio da relação pedagógica que envolva intencionalidades de ação, objetivadas em condutas, atitudes e habilidades de docentes e discentes. Na condição de avaliador desse processo, o professor interpreta e atribui sentidos e significados à avaliação, produzindo conhecimentos e representações a respeito da avaliação e acerca de seu papel como avaliador, com base em suas próprias concepções, vivências e conhecimentos (SORDI, 2001; CHUEIRI, 2008).

O trabalho de Bloom; Hastings; Madaus (1971), no "Manual de Avaliação Formativa e Somativa do Aprendizado Escolar", foi o primeiro a discutir múltiplas estratégias de avaliação, dividindo-as em diagnóstica, formativa e somativa. A avaliação diagnóstica tem como objetivo verificar se os discentes possuem os conhecimentos e habilidades imprescindíveis para as novas aprendizagens, devendo ser realizada no início do processo. A formativa é a que ocorre ao longo do processo de aprendizagem, seu objetivo é a correção de falhas do processo educacional e a prescrição de medidas alternativas de recuperação dessas falhas, e por fim avaliação somativa ocorre ao final de um processo, com claros objetivos de mensuração de resultados.

É importante salientar que uma avaliação é realmente formativa se, através dela, os discentes se conscientizarem das eventuais diferenças entre o seu estado presente e o que se deve alcançar e do que é necessário fazer para reduzir ou mesmo eliminar tais diferenças. Desta forma, não basta apenas realizar avaliações em vários momentos do processo de aprendizagem; uma vez que uma avaliação pode ser contínua sem ser formativa (PERRENOUD, 1999; MASETTO, 2003; FERNANDES, 2008).

A avaliação é o processo central de um ensino eficaz e, é por meio dela que podemos descobrir se o planejamento de atividades propostas de ensino resultou em aprendizagem. Uma avaliação de alta qualidade é necessária para interpretar, de forma satisfatória, o que os examinados sabem e o que são capazes de fazer com relação ao domínio-alvo. Técnicas de avaliação tradicionais, como os exames de múltipla escolha e verdadeiro-falso, fazem muito pouco para avaliar verdadeiramente a compreensão do discente e do aprendizado contínuo na experiência em metodologias ativas, exigindo formas de avaliação mais complexas (BARROWS; TAMBLYN, 1980; BIRENBAUM, 2007; WILIAM, 2011). 
Refletindo sobre como se dá o processo de construção do conhecimento em metodologias ativas de ensino, é fundamental que a avaliação seja constantemente discutida, já que mudar e melhorar práticas de avaliação implica que o seu significado seja claro para os diferentes intervenientes e, muito particularmente, para os professores e investigadores. As ações que propiciem o caminhar em direção a um processo mais justo e frutífero de avaliação devem ser buscadas e introduzidas de maneira efetiva, aspirando-se conseguir, mesmo que lentamente, a almejada mudança. Para isso necessita-se de pesquisas que verifiquem a eficácia das práticas de avaliação formativa em relação à avaliação somativa utilizada (GULIKERS et al., 2013).

Diante do cenário descrito e da ausência de estudos a respeito, os objetivos deste estudo foram comparar e verificar a relação entre os resultados das avaliações formativas e somativas em duas atividades, as Sessões Tutoriais e Habilidades Práticas de estudantes de um Curso de Graduação de uma universidade pública brasileira.

\section{MÉTODO}

Trata-se de um estudo observacional, com abordagem quantitativa, analítica e transversal. O estudo foi aprovado pelo Comitê de Ética em Pesquisa por meio da Plataforma Brasil, com número CAAE 33665414.6.0000.5546, cumprindo com o determinado na Resolução do Conselho Nacional de Saúde nº 466/12.

\section{Amostra}

Para compor a amostra do estudo foram analisadas as notas obtidas de avaliações modulares somativas e formativas de 42 estudantes de uma Universidade pública que tem como método de ensino-aprendizagem a metodologia ativa, com a abordagem da aprendizagem baseada em problemas.

Para a inclusão na amostra foram selecionadas apenas as avaliações do II Ciclo do curso de graduação em Fonoaudiologia. Foram incluídas as avaliações de discentes que apresentaram $75 \%$ ou mais de assiduidade. Foram eleitos os módulos profissionalizantes e obrigatórios deste Ciclo, ou seja, o bloco temático de Infância e Adolescência. Este bloco ocorre anualmente e possui como foco a atenção primária à saúde e nível complementar da atenção básica, no qual são abordados os temas de 
estudo da linguagem infantil; voz na criança e adolescente; sucção, respiração, deglutição e mastigação; audição na criança e adolescência; habilidades fonoaudiológicas e práticas de laboratório. Assim, há atividades caracterizadas como sessões tutoriais e habilidades práticas.

Foram excluídos da amostra avaliações do I Ciclo do curso de graduação em Fonoaudiologia, por se tratar de um ciclo comum para todos os estudantes da área de saúde. Excluiu-se também avaliações do III e IV Ciclo por não se tratarem do mesmo bloco temático de estudo, e também, os estudantes que no decorrer do percurso acadêmico no respectivo Ciclo, desistiram do Curso ou excederam o limite de faltas de $25 \%$.

\section{Procedimentos}

As avaliações somativas e formativas do II Ciclo do curso de graduação em Fonoaudiologia foram capturadas via registro acadêmico pelos docentes na plataforma SIGAA - Sistema Integrado de Gestão de Atividades Acadêmicas, no qual foram obtidas as notas das sessões tutoriais e habilidades práticas.

Nas sessões tutoriais, os conteúdos de cada módulo são distribuídos em nove problemas que são trabalhados em dez sessões, na frequência de duas vezes por semana, sendo três horas/aula cada. A avaliação somativa é realizada por meio de uma prova teórica, contextualizada com os problemas abordados nas sessões tutoriais, de forma a simular as situações reais. A avaliação formativa avalia o desempenho do estudante diariamente, por meio de critérios elaborados pelo Núcleo Docente Estruturante, Colegiado do Curso e Centro Acadêmico. Dessa forma, diariamente, diante das situações-problemas discutidas na sessão de tutorial, o tutor realiza a avaliação formativa, considerando os seguintes critérios de avaliação:

1. Na abertura da situação-problema: o discente é avaliado quanto a habilidade em identificar questões, utilização de conhecimentos prévios, capacidade de gerar hipóteses e se demonstra capacidade de sintetizar e expor ideias de forma clara e organizada.

2. No fechamento da situação-problema: avalia-se se o discente demonstrou estudo prévio, trazendo informações pertinentes aos objetivos propostos, se é capaz de sintetizar e expor informações de forma clara e organizada e se apresenta atitude crítica em relação às informações trazidas. 
3. Na interação com o grupo, tanto na abertura quanto no fechamento da situação-problema, verifica-se a pontualidade, se é capaz de desempenhar seu papel no grupo, se apresenta relacionamento interpessoal satisfatório com o tutor e demais membros do grupo, bem como se é capaz de criticar e receber críticas.

É importante ressaltar que no presente estudo foram capturadas apenas as notas diárias da avaliação formativas atribuídas pelo tutor, mediante aos critérios expostos acima. Desta forma, não foram consideradas as demais avaliações somativas, tais como notas de portfólio, autoavaliações e avaliações interpares.

Nas habilidades práticas, o conteúdo de cada módulo é dividido em seis encontros com quatro horas/aula, na frequência de uma vez por semana. Para o presente estudo foram consideradas as notas diárias atribuída pelo tutor na avaliação formativa, sendo considerados os seguintes critérios:

1. Aspectos técnicos: verifica-se o conhecimento prático demonstrado nas atividades práticas desenvolvidas, conhecimento teórico e a resolução de exercícios com associação teórico-prática.

2. Aspectos pessoais: verifica-se a assiduidade e pontualidade, responsabilidade, disciplina, condutas éticas.

A avaliação somativa é realizada por meio de uma prova, de caráter teóricoprático, contextualizada às atividades realizadas na prática de habilidades e de forma a simular as situações reais.

As notas obtidas das avaliações formativa e somativa das sessões tutorias e habilidades práticas foram expressas em escala numérica de zero a dez e foram tabuladas em planilha Excel (Microsoft Office®) e submetidas ao tratamento estatístico por meio de software pelo SPSS® 15.0 para o Windows (SPSS Inc., 1989-2006, Chicago, Illinois, EUA) - Ribas; Vieira (2011).

Primeiramente, realizou-se a análise descritiva dos dados. Em seguida, as médias e desvios padrões foram apresentados na forma de gráficos e tabelas, sendo submetidos a testes estatísticos não paramétricos.

Foi utilizado o teste de correlação bivariada, com coeficiente de Spearman, para correlacionar as notas dos discentes nas avaliações somativas e formativas, bem como o teste Wilcoxon, para a comparação entre as médias das respectivas avaliações nas sessões tutoriais e nas habilidades práticas. Para todos os testes foi considerado grau de significância de $5 \%$ (p valor $<0,05)$. 


\section{Resultados}

A amostra foi composta por resultados das avaliações de 42 estudantes, com faixa etária entre 18 e 39 anos, média de 23 anos \pm 5 , sendo a maioria do gênero feminino $(80,95 \%)$.

Observou-se, como pode ser visualizado no Gráfico 1, maiores valores para as médias de avaliação formativa, quando comparada à somativa, independentemente de ser uma atividade de tutorial ou habilidades práticas.

Gráfico 1: Valores médios das notas das avaliações formativa e somativa nos grupos tutoriais e habilidades de 42 discentes de um curso de graduação em Fonoaudiologia

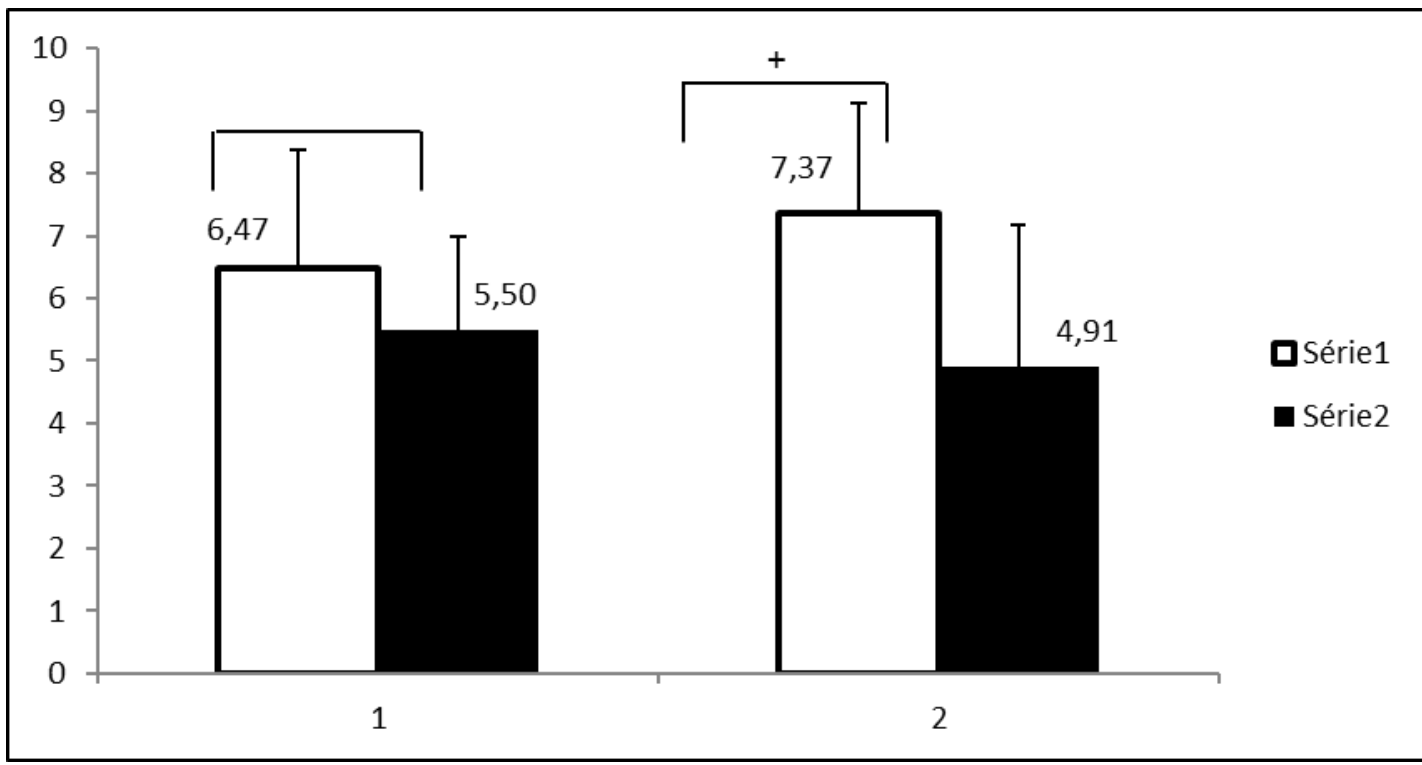

Legenda: * $\mathrm{e}^{+}$indicam diferença estatística por meio do teste de Wilcoxon $(\mathrm{p}<0,01)$. Fonte: Elaboração própria a partir de dados da pesquisa.

Por meio do coeficiente de Spearman, foi verificada correlação positiva (leve/moderada) entre as notas referentes às avaliações formativas e somativas ( $p$ $<0,01)$, tanto na subunidade de tutorial $(R=0,39)$, quanto de habilidades $(R=0,46)$. Ao comparar as notas das avaliações formativas com a avaliação somativa, utilizando-se o Teste de Wilcoxon, constatou-se presença de diferença significativa para tutorial e para habilidades, com p valores menores que 0,01 .

A avaliação formativa foi mais bem pontuada pelos discentes nas atividades de Habilidades Práticas e a somativa nas Sessões Tutoriais, com presença de diferença significativa entre o mesmo tipo de avaliação ( $\mathrm{p}<0,05$ - Teste de Mann Whitney). 


\section{Discussão}

O uso de metodologias ativas de aprendizagem considera a estrutura lógica de pensamento do estudante para que construa o conhecimento através do desenvolvimento de seu raciocínio crítico (FREIRE, 2006; GWEE, 2008).

Diante do uso dessas metodologias como método de ensino-aprendizagem, devese repensar o processo de avaliação, uma vez que esta tem um papel muito importante, o papel formativo. Não deve ser compreendida como forma de julgamento, punição ou de classificação. Avaliar faz parte da formação, envolve diversos sujeitos e deve servir para o desenvolvimento e para a construção de novos conhecimentos (FROTA et al., 2011; WILIAM, 2011).

Desta maneira, o Curso de Fonoaudiologia, cenário do presente estudo, ao adotar o método $\mathrm{ABP}$, realizou a implementação de mudanças metodológicas, pautadas em uma discussão sobre as demandas surgidas nos processos de reestruturação de currículos (DOCHY, 2001; VILLAS-BOAS, 2005; MENDOZA, 2012, DORNELAS et al., 2014). Ao elaborar o processo de avaliação buscou-se apreender a essência do desenvolvimento de cada educando em seu processo de formação, permitindo a expressão da subjetividade e da busca do conhecimento construído, apreendido e ressignificado pelo estudante, em um espiral crescente de envolvimento e desenvolvimento técnico-científico (FREIRE, 2006; BENNETT, 2009; FROTA et al., 2011; DORNELAS et al., 2016).

Pensando a partir desta perspectiva, foram escolhidos instrumentos de avaliação em consonância com os eixos trabalhados ao longo da formação em Saúde, os quais deveriam oportunizar aos tutores e instrutores, discentes e à própria instituição, um momento de reflexão sobre as práticas desenvolvidas e desempenhos alcançados, bem como a elaboração de estratégias para o aperfeiçoamento da aprendizagem (WILIAM et al, 2004; COSTA, 2011; WILIAM, 2011; LIMBERGER, 2013).

Neste direcionamento, utilizam-se métodos de avaliação não apenas de maneira somativa (ao final do processo de ensino/aprendizagem); mas também formativa, durante esse processo, como, por exemplo, por meio da observação dos estudantes, das perguntas, das respostas aos questionamentos, da elaboração de sínteses orais e escritas, com o uso de portfólios reflexivos (BIRENBAUM, 2007; GULIKERS et al., 2013). 
No curso em questão, a avaliação formativa foi construída de forma a, diariamente, auxiliar o estudante a aprimorar suas habilidades de comunicação (oral e escrita), liderança, relacionamento interpessoal, colaboração, sendo instigado a utilizar seus conhecimentos prévios, a gerar hipóteses, a analisar, sintetizar, discutir e construir pensamento crítico a respeito das informações técnico-científicas da área, das suas atitudes enquanto corresponsável pela sua própria formação e a de seus colegas. Por outro lado, o tutor, que é um profissional formado em metodologia tradicional, precisa se deslocar de seu papel centralizador, para se colocar em uma posição de mediador do conhecimento e, desta forma, rever o processo de avaliação, que vai além de uma avaliação somativa, considerando o processo, o percurso percorrido pelo estudante.

No presente estudo foi observado maiores escores nas avaliações formativas quando comparadas às somativas (Gráfico 1). Bennett (2009) apontou que o conceito de avaliação somativa vai depender dos significados atribuídos e das estratégias utilizadas por cada Instituição. Assim, no presente estudo, os achados podem ser justificados pelas características das provas utilizadas, as quais exigiam raciocínio lógico e não apenas atividades que objetivavam analisar a memorização de conteúdo, o que se diferencia das provas tradicionais, difundidas como a principal estratégia de avaliação somativa (MATHEMATICAL SCIENCES EDUCATION BOARD, 1993).

Nesta mesma vertente, Gulikers et al. (2013) salientou que é um problema bastante comum encontrar ausência de alinhamento entre práticas de avaliação somativa e formativa, em que a avaliação somativa é algo com maior padronização educacional, enquanto as práticas de avaliação formativa são de responsabilidade de cada escola ou mesmo da ação individual de professores.

Associado a esses achados, as notas da avaliação formativa, ao se correlacionarem positivamente com as da somativa (de forma leve/moderada), demonstram que estas avaliações diárias podem ser utilizadas como guia do processo de aprendizado, além de contribuir para a reorientação e ajustes metodológicos no processo de ensino (RUIZ-PRIMO, 2011; GULIKERS et al., 2013).

Wiliam et al. (2004) verificaram que as estratégias utilizadas na avaliação formativa aumentam a taxa de aprendizagem dos estudantes em $70 \%$, ou um extra de oito meses de aprendizagem por ano. Concordamos com o exposto, pois no curso em questão são utilizadas diferentes estratégias que exigem habilidades comunicativas orais e escritas, bem como a assumpção de diferentes papéis (membro de um grupo, secretário ou coordenador). 
O fato das avaliações formativas, em todos os achados, apresentarem maiores médias do que as somativas pode estar relacionado a um maior estímulo do discente ao se encontrar diante de atividades que "simulam situações reais" de sua futura prática clínica, e é justificado como produto de uma aprendizagem significativa, ou seja, aquela que faz sentido e possui aplicabilidade e relevância teórico-prática. Além disso, os estudantes costumam designar novas formas de avaliação como interessantes e motivadoras (DOCHY, 2001).

Esta diferença pode ser também justificada pelo fato do discente inserido em um curso com metodologia ativa apresentar uma maior facilidade de expressão oral, de busca ativa e de resolução frente a um problema exposto do que quando a avaliação é realizada de forma tradicional. Mesmo com todos os cuidados para que a avaliação somativa se aproxime da prática profissional, existe, neste espaço, a sensação desconfortável de ser avaliado e a necessidade de se mostrar um determinado conhecimento, o que contribui, sem dúvidas, para a diferença significante entre o desempenho dos discentes nas duas formas de avaliação.

Em contrapartida, a avaliação formativa ocorre de forma contextualizada e dentro do processo didático, no ambiente de sala de aula, em que a relação tutordiscente é mais próxima. Esta prática avaliativa permite que o tutor observe, registre e aja frente aos indícios das dificuldades apresentadas pelos discentes durante a aprendizagem (ALVARENGA, 2001), além de ser processual, permitindo reflexão do desempenho, por parte do discente, que pode modificar sua atuação em próxima sessão tutorial - o que já não ocorre em avaliações somativas, por sua própria definição.

Ainda pode ser observado no Gráfico 1, que ao se comparar a avaliação formativa, tanto no Tutorial quanto em Habilidades, os discentes apresentaram média de notas maior em Habilidades. Em contrapartida, ao se comparar as médias de notas da avaliação somativa, o melhor desempenho discente ocorreu nos Tutoriais. Tais resultados demonstram que as estratégias de ensino e aprendizagem refletem suas formas de avaliação, uma vez que nos Tutoriais as discussões versam sobre o que a literatura comenta sobre determinado assunto, sendo mais teórico, e nas Habilidades, os instrumentos clínico-terapêuticos são manuseados, exercícios são realizados e a prática é discutida, sem deixar a teoria de lado, mas privilegiando a prática. Além disso, há que se discutir o interesse do aluno pelos aspectos clínico-terapêuticos contemplados principalmente nas Habilidades. Sem dúvidas, os resultados demonstram que alguns 
ajustes serão necessários para que a distância entre os conhecimentos teóricos e práticos diminuam.

É fundamental estimular, desde o início da graduação, a articulação entre teoria e prática, a fim de possibilitar aos estudantes, de forma contextualizada, a construção de conhecimentos para uma formação comprometida social e eticamente (FRANCO; BOOG, 2007). Daí a importância da avaliação formativa no âmbito de Cursos inseridos em metodologias ativas.

Faz-se ímpar ressaltar que ainda se faz necessária a discussão a respeito da formação docente em Saúde, da concepção docente do que é considerado como atividade teórico-prática e de como realizar avaliações que contemplem situações do cotidiano profissional (FRANCO; BOOG, 2007) e que revelem uma prática baseada em evidências técnico-científicas, independente da subunidade curricular cursada pelo discente. Tarefa esta que só se faz quando o corpo docente se sente comprometido com a formação estudantil, analisando os resultados obtidos em prol de melhorias no processo de ensino e aprendizagem.

\section{Conclusão}

Conclui-se que houve correlação positiva entre as avaliações formativas e somativas em Tutoriais e Habilidades, com melhores resultados para a formativa (tanto em Tutoriais quanto em Habilidades).

Diante desses resultados, pode-se afirmar que o processo percorrido pelo discente do referido curso tem sido valorizado pelo corpo docente, embora se reforce a necessidade de aprender a avaliar, a encarar este momento como realmente um momento potencializador, regulador da aprendizagem, no sentido de integrar, de propor caminhos a serem percorridos, mas não o caminho com metas definidas, estabelecidas sem espaço para discussões, paradas e descobertas.

Sentimos a necessidade de o professor reformular suas práticas constantemente, refletindo-as de forma que compreenda o dinamismo das relações intrinsicamente existentes nesse processo. Se o que se almeja em uma metodologia ativa de ensino é a formação de sujeitos críticos, os docentes envolvidos neste processo também o devem ser. 


\section{Referências}

ALVAREnGA, G. M. Portfólio: o que é e a que serve? Revista Olho Mágico, Londrina, v. 8, n. 1, p. 19-21, 2001.

BARROWS, H.; TAMBLYN, R. M. Problem-based learning: an approach to medical education. New York: Springer Publishing Company, 1980.

BENNETT, R. E. A critical look at the meaning and basis of formative assessment. Princeton: Educational Testing Service, 2009.

BERBEL, N. A. N. A problematização e a aprendizagem baseada em problemas: diferentes termos ou diferentes caminhos? Interface - Comunicação, Saúde, Educação, Botucatu, v. 2, n. 2, p. 139-154, 1998.

BIRENBAUM, M. Evaluating the assessment: sources of evidence for quality assurance. Studies in Educational Evaluation, Amsterdam, v. 33, p. 29-49, 2007.

BLOOM, B. S.; HASTINGS, J. T.; MADAUS, G. F.; FLOREZ, M. C. F.; PATTO, M. H. S.; QUINTÃO, L. R.; VANZOLINI, M. E. Manual de avaliação formativa e somativa do aprendizado escolar. São Paulo: Pioneira, 1971.

BORGES, M. C.; CHACHÁ, S. G. F.; QUINTANA, S. M.; FREITAS, L. C. C.; RODRIGUES, M.L.V. Aprendizado baseado em problemas. Revista de Medicina, Ribeirão Preto, vol. 47, n.3, p. 301-307, 2014.

CYRINO, E. G.; TORALLES-PEREIRA, M. L. Trabalhando com estratégias de ensinoaprendizado por descoberta na área da saúde: a problematização e a aprendizagem baseada em problemas. Cadernos de Saúde Pública, Rio de Janeiro, v. 20, n. 3, p. 780-788, 2004.

CHUEIRI, M.S. F. Concepções sobre a avaliação escolar. Estudos em avaliação educacional, São Paulo, v. 19, p. 39, 49-64, jan. abr. 2008.

COSTA, E. M. M. B. C. Avaliação da aprendizagem: da teoria à prática. In: CECY, C.; OLIVEIRA, G. A.; COSTA, E. M. M. B. C. (Org.). Melhoria da qualidade da educação farmacêutica. Brasília: Associação Brasileira de Ensino Farmacêutico e Bioquímico, p. 111-128, 2011.

DOCHY, F. A new assessment era: different needs, new challenges. Learning and Instruction, Amsterdam, v. 10, p. 11-20, 2001.

DORNELAS R.; BARRETO, A.C.O.; GRANZOTTI, R.B.G.; DOMENIS, D.R.; CESAR, C.P.H.A.R.; ALENCAR, R.I.; SILVA, K. Metodologias Ativas: Uma experiência na Fonoaudiologia. Distúrbios da Comunicação, v. 26, n. 4, p. 815-21, 2014.

DORNELAS, R.; SILVA, K.; CESAR, C. P. H. A. R.; OLIVEIRA-BARRETO, A. C.; GUEDES-GRANZOTTI, R. B. Avaliação da situação-problema por tutores e discentes em um curso de graduação vivenciado em metodologias ativas. Revista IberoAmericana de Estudos em Educação, Araraquara, v. 11, n. 1, p. 245-257, 2016. 
FERNANDES, D. Para uma teoria da avaliação no domínio das aprendizagens. Estudos em Avaliação Educacional, São Paulo, v. 19, n. 41, p. 347-372, set. dez. 2008.

FRANCO, A. C.; BOOG, M. C. F. Relação teoria-prática no ensino de educação nutricional. Revista Nutr., Campinas, v.20, n.6, p.643-655, 2007. Disponível em: <http://www.scielo.br/pdf/rn/v20n6/a07v20n6.pdf>. Acesso em: 10 abr. 2014.

FREIRE, P. Pedagogia da autonomia: saberes necessários à prática educativa. 33. ed. São Paulo: Paz e Terra, 2006.

FROTA, M. M. A.; MENEZES, L. M. B.; ALENCAR, C. H.; JORGE, L. S.; ALMEIDA, M. E. L. O portfólio como estratégia facilitadora do processo de ensinoaprendizagem para a formação em odontologia. Adequação de metodologias de ensino utilizando o ambiente virtual de aprendizagem. Revista da ABENO, Brasília, v. 11, n. 1, p. 23-28, 2011.

GARCIA MADRUGA, J. A. Aprendizagem pela descoberta frente à aprendizagem pela recepção: a teoria da aprendizagem verbal significativa. In: COLL, C.; PALÁCIOS, J.; MARCHESI, A. (Orgs.). Desenvolvimento psicológico e educação. Porto Alegre: Artmed, p. 68-78, 1996.

GUEDES-GRANZOTTI, R. B.; SILVA, K.; DORNELAS, R.; CESAR, C. P. H. A. R.; PELLICANI, A. D.; DOMENIS, D. R. Situação-Problemas como disparador do processo de ensino-aprendizagem em metodologias ativas de ensino. Revista Cefac. Vol. 17, n. 6, p. 2081-2087, 2015.

GULIKERS, J. T.M.; BIEMANS, H. J. A.; WESSELINK, R.; VAN DER WEL, M. Aligning formative and summative assessments: a collaborative action research challenging teacher conceptions. Studies in Educational Evaluation, Amsterdam,v. 39, p. 116-124, 2013.

GWEE, M. C. E. Globalization of problem-based learning (pbl): cross-cultural implications. Kaohsiung J Med Sci, Taiwan, v. 24, n. 3 (suppl.), p. S14-S22, March 2008.

LIMBERGER, J. B. Metodologias ativas de ensino-aprendizagem para educação farmacêutica: um relato de experiência. Comunicação, Saúde, Educação, Botucatu, v. 17, n.47, p. 969-975, out. dez. 2013.

MARIN, M. J. S. et al. Pós-graduação multiprofissional em saúde: resultados de experiências utilizando metodologias ativas. Interface, Comunicação, Saúde, Educação, Botucatu, v. 14, n. 33, p. 331-344, 2010.

MASETTO, M. Competência pedagógica do professor universitário. São Paulo: Summus, 2003.

MATHEMATICAL SCIENCES EDUCATION BOARD. Measuring what counts, a conceptual guide for mathematics assessment. Washington: National Academy Press, 1993.

MENDOZA, V. L. F. Avaliação da aprendizagem nos cursos da área de saúde. Revista Matogrossense de Enfermagem, Mato Grosso, v. 1, n. 2, p. 183-191, nov. dez. 2012. 
MERHY, E. E.; FEUERWERKER, L. C. M.; CECCIM, R. B. Educación permanente en salud: una estrategia para intervenir en la micropolítica del trabajo en salud. Salud Colectiva, La Plata, v. 2, n. 2, p. 147-160, 2006.

PERRENOUD, P. Avaliação: da excelência à regulação das aprendizagens - entre duas lógicas. São Paulo: Artmed, 1999.

RIBAS, J. R.; VIEIRA, P. R. C. Análise multivariada com o uso do SPSS. Rio de Janeiro: Ciência Moderna, 2011.

RUIZ-PRIMO, M. A. Informal formative assessment: the role of instructional dialogues in assessing students' learning. Studies in Educational Evaluation, Amsterdam, v. 37, p. 15-24, 2011.

SORDI, M. R. L. Alternativas propositivas no campo da avaliação: por que não? In: CASTANHO S.; CASTANHO, M. E. (Orgs.). Temas e textos em metodologia do ensino superior. Campinas: Papirus, p. 171-182, 2001.

VILLAS-BOAS, B. M. F. O portfólio no curso de Pedagogia: ampliando o diálogo entre professores e aluno. Educ Soc., Campinas, v. 26, n. 90, p. 291-306, 2005.

ZANOTTO, M.; ROSE, T. Problematizar a própria realidade: análise de uma experiência de formação contínua. Educação e Pesquisa, São Paulo, v. 29, n. 1, p. 4554, 2003.

WILIAM, D. What is assessment for learning? Studies in Educational Evaluation, Amsterdam, v. 37, p. 3-14, 2011.

WILIAM, D.; LEE, C.; HARRISON, C; BLACK, P. Teachers developing assessment for learning: impact on student achievement. Assessment in Education, London, v. 11, n. 1, p. 49-65, March 2004.

\section{Como referenciar este artigo:}

OLIVEIRA-BARRETO, Aline Cabral de et al. Métodos de avaliação discente em um curso de graduação baseado em metodologias ativas. Revista Ibero-Americana de Estudos em Educação, Araraquara, v. 12, n. 2, p. 1005-1019, 2017. Disponível em: <http://dx.doi.org/10.21723/riaee.v12.n2.8745>. E-ISSN: 1982-5587.

Submetido em: 30/05/2016

Aprovação final em: 28/03/2017 